\title{
Positive-Overlap Transition and Critical Exponents in Mean Field Spin Glasses
}

\author{
Alessandra Agostini *, Adriano Barra ${ }^{\dagger}$ Luca De Sanctis $\ddagger$
}

May 2, 2017

\begin{abstract}
In this paper we obtain two results for the Sherrington-Kirkpatrick (SK) model, and we show that they both emerge from a single approach. First, we prove that the average of the overlap takes positive values when it is non zero. More specificly, the average of the overlap, which is naively expected to take values in the whole interval $[-1,+1]$, becomes positive if we "first" apply an external field, so to destroy the gauge invariance of the model, and "then" remove it in the thermodynamic limit. This phenomenon emerges at the critical point. This first result is weaker than the one obtained by Talagrand (not limited to the average of the overlap), but we show here that, at least in average, the overlap is proven to be non-negative with no use of the Ghirlanda-Guerra identities. The latter are instead needed to obtain the second result, which is the control the behavior of the
\end{abstract}

${ }^{*}$ School of Mathematics, Cardiff University, Senghennydd Road, Cardiff, CF24 4AG, Wales, United Kingdom <agostinia@cf .ac.uk>

${ }^{\dagger}$ King's College London, Department of Mathematics, Strand, London WC2R 2LS, United Kingdom, and Dipartimento di Fisica, Università di Roma "La Sapienza" Piazzale Aldo Moro 2, 00185 Roma, Italy, <Adriano.Barra@roma1.infn.it>

${ }^{\ddagger}$ ICTP, Strada Costiera 11, 34014 Trieste, Italy, <lde_sanc@ictp.it> 
overlap at the critical point: we find the critical exponents of all the overlap correlation functions.

\section{Introduction}

Mean field spin glass models are a very hard "challenge for mathematicians" [11. In fact, despite the important fact that the free energy is known (predicted by Parisi and rigorously proven by Talagrand), very little is proven about their organization into pure states at low temperature except for high temperature regimes. Only a sequence of brilliant intuitions of Parisi (see [9] and references therein) provided a proposal for a theory of disordered systems, which is comforted by many numerical studies (see for example [8]), but many of the intuitions still lack a rigorous proof. Among the many stimulating suggestions, Parisi prescribed the positivity of the overlap below the critical temperature, which breaks the symmetry by which the overlap should be trivially zero (in absence of an external field). More precisely, the average of the overlap is zero when there is no external field, by spin-flip symmetry. If an external field is applied, the spin-flip symmetry is broken and the average of the overlap is non-zero. When the external field is removed the average of the overlap remains strictly positive, below the critical temperature. This is what we prove here (slightly more, to be precise, as we will use a random external field as opposed to a constant one) in agreement with what Parisi predicted. This result is weaker than what Talagrand proved [11, namely that the overlap is non-negative with the possible exception of a zero Lebesgue measure set of the parameters. This is shown to be a consequence of the GhirlandaGuerra identities [4. Here we show that at least the weaker result can be proven in a "very physical" and simple way. Moreover, the technique offers another result, of crucial physical relevance, i.e. the critical exponents of the behavior of the overlaps at the critical point.

We will focus on the following points 
1. spin-flip symmetry breaking due to an external field;

2. strict positivity of the average of the overlap below the critical temperature;

3. gauge symmetry breaking due to an external field;

4. critical exponents in connection with the gauge symmetry breaking.

The procedure we employed to prove these results makes use of an iterative approach to expand the average of the overlap in a power series of the strength of the external field. Within a framework typical of cavity approaches, we also take advantage of some known symmetries of the model, which we report together with the definition of the model in section 2 , The main results are presented in sections 3 and 4 , where the positivity of the overlap and the critical exponents are studied respectively. Conclusive remarks are left for section 5

\section{Model, notations, previous results}

The Sherrington-Kirkpatrick (SK) model describes a system of $N$ binary spins $\sigma_{i}, i \in(1, \ldots, N)$. A configuration $\sigma$ of the system is then a map

$$
\sigma:\{1,2, \ldots, N\} \ni i \rightarrow \sigma_{i} \in\{-1,+1\} \text {. }
$$

The Hamiltonian of the model is defined to assign the following energy to a given configuration $\sigma$ :

$$
H_{N}(\sigma ; J)=\frac{-1}{\sqrt{N}} \sum_{(i, j)} J_{i j} \sigma_{i} \sigma_{j}
$$

where the sum ranges over all the $N(N-1) / 2$ different couples of indices $(i, j)$, and $J_{i j}$ are independ centered unit Gaussian random variables. The partition function is defined as usual by

$$
Z_{N}(\beta ; J)=\sum_{\sigma} \exp (-\beta H(\sigma ; J))
$$


while the Boltzmann-Gibbs expectation of an observable $A: \sigma \rightarrow \mathbb{R}$ is

$$
\omega(A)=\frac{1}{Z_{N}(\beta ; J)} \sum_{\sigma} A(\sigma) \exp (-\beta H(\beta ; J)) .
$$

The global average (over the spins first and the noise in the couplings afterwards) is $\langle\cdot\rangle=\mathbb{E} \omega(\cdot)$, where $\mathbb{E}$ denotes the expectation with respect to all the (quenched) Gaussian variables. The notation for the averages does not specify any dependence on the size of the system, and we will occasionally use the same symbols in the thermodynamic limit too, specifying this when needed. By $\Omega$ we denote the product measure (replica measure) of the needed number of copies of $\omega$, which we will use when dealing with functions of several configurations (replicas). Notice that while $\Omega$ is factorized by definition, $\langle\cdot\rangle$ is not, as we are just replicating configurations, keeping for each the same disorder (i.e. Gaussian couplings). Taking the same disorder results in coupling the various replicas and therefore $\mathbb{E}$ destroys the factorization. Given two replicas $\sigma^{(1)}$ and $\sigma^{(2)}$ we define the overlap between them as

$$
q_{12}=\frac{1}{N} \sum_{i=1}^{N} \sigma_{i}^{(1)} \sigma_{i}^{(2)}
$$

sometimes simply written as $q$. The pressure $\alpha_{N}(\beta)$ and the free energy per spin $f_{N}(\beta)$ are defined as

$$
\alpha_{N}(\beta)=-\beta f_{N}(\beta)=\frac{1}{N} \mathbb{E} \ln Z_{N}(\beta) .
$$

By a trivial (spin-flip) symmetry argument, one readily sees that (for all inverse temperature $\beta$ )

$$
q_{12} \in[-1,1],\left\langle q_{12}\right\rangle=0 .
$$

When an external field is applied, i.e. a term of the form $\sqrt{x} \sum_{i=1}^{N} J_{i} \sigma_{i}$ for $J \in \mathcal{N}(0,1)$ and $x \in \mathbb{R}_{+}$is added to the Hamiltonian, the spin-flip symmetry is broken and the overlap will be different from zero in average. 
Parisi theory [9] prescribes a phase transition such that even an infinitesimal external field will force the overlap to be supported on $[0,1]$, and the limit as $x \rightarrow 0^{+}$of the average of the overlap is positive:

$$
\lim _{x \rightarrow 0^{+}}\left\langle q_{12}\right\rangle>0, \beta>\beta_{c}
$$

where $\beta_{c}$ is the critical inverse temperature and clearly the dependence on $x$ of the measure $\langle\cdot\rangle=\mathbb{E} \omega(\cdot)$ is here in the Boltzmannfaktor in $\omega$, which includes the external field term in addition to the original Hamiltonian. For the sake of clarity, we will sometimes attach a sub $x$ to the measures, when they include the external field.

\subsection{Some useful results}

The next two propositions, which we do not provide the easy proof of, will be of precious help.

Proposition 1 The average $\langle\cdot\rangle$ is invariant under replica symmetry: let $g$ be any element of the group $\mathbf{G}$ of permutations of s objects, then

$$
\left\langle\phi\left(q_{a b}\right)\right\rangle=\left\langle\phi\left(q_{g(a) g(b)}\right)\right\rangle
$$

for any function $\phi$ of two generic replicas and all $a, b \in\{1, \ldots, s\}$.

Proposition 2 The average $\langle\cdot\rangle$ is invariant under gauge symmetry:

$$
\sigma_{i}^{a} \rightarrow \epsilon_{a} \sigma_{i}^{a}, q_{a b} \rightarrow \epsilon_{a} \epsilon_{b} q_{a b}
$$

being $\epsilon_{a}, \epsilon_{b}= \pm 1$.

This symmetry is a consequence of the parity of the state $\omega$ and of the dichotomic nature of the spin, so it is obviously guaranteed by the spin-flip symmetry of the Hamiltonian for each single replica in $\Omega$.

We will have to compute several averages of polynomials of overlaps, but will see that the average of certain polynomials are easy to compute. That is why the following classification comes in handy. 
Definition 1 A polynomial function of some overlaps is said

- filled if every replica appears an even number of times in it;

- fillable if it can be made filled multiplying it by exactly one overlap of two replicas;

- unfillable if it is neither filled nor fillable.

A consequence of the gauge invariance of the model is that the average of non filled polynomials (fillable as well as unfillable) is identically zero. The reason is that the absence of an external field guarantees gauge invariance, and non-filled polynomials are asymmetric in one of the overlaps and averages of products of spins belonging to different replicas are zero by the symmetries above. Consequently, in this case the probability distribution of functions of overlaps is uniquely determined by its restriction to the positive semi-domain $[0,1]$. The general case, at zero external field, in which $q \in[-1,+1]$, is obtainable simply by employing the gauge.

\section{Discontinuity of the order parameter}

In order to establish the presence of a discontinuity in the overlap due to an infinitesimal external field, we employ some kind of cavity method and stochastic stability techniques. Namely, we add a random perturbation (as the one described above) which can be equivalent either to a deterministic

external field or to the addition of a new spin (depending on the choice of the parameters), and we then study the consequences of the spontaneous breaking of the spin-flip and gauge symmetries.

\subsection{The external field and non-negativity of the aver- age of the overlap}

Before proceeding, in the next subsection, with the introduction of the main techniques, let us introduce the needed external field which breaks the spin- 
flip symmetry of the Hamiltonian and hence of $\omega$. Consider a family $\left\{J_{i}\right\}$ of $N$ centered unit Gaussian independent random variables. The expectation with respect to these (quenched) variables will be included in $\mathbb{E}$. Let us introduce consequently the following Boltzmannfaktor

$$
B(x)=\exp \left(-\beta H_{N}(\sigma ; J)+\sqrt{x} \sum_{i=1}^{N} J_{i} \sigma_{i}\right) .
$$

We will here denote the corresponding Gibbs measure by $\omega_{x}$, and $\langle\cdot\rangle_{x} \equiv$ $\mathbb{E} \omega_{x}(\cdot)$. In the following we will be interested in letting $x$ go to zero as $N^{-1}$ while $N$ goes to infinity. This is the only way to recover the correct normalization for a Hamiltonian of $N+1$ spins (that is the proper weight of the Boltzmann factor $B(x)$ if the gauge symmetry holds), and it is what is technically needed to get our main result, as it will emerge.

Let us now report a result that generalizes slightly the analogous one proven in [2].

Proposition 3 In the $N \rightarrow \infty$ limit the average $\langle\cdot\rangle_{x}$ of filled polynomials is $x$-independent, i.e. $\left\langle q_{12} q_{23} q_{13}\right\rangle_{x}=\left\langle q_{12} q_{23} q_{13}\right\rangle$, when integrated over any interval $\left[\beta_{1}, \beta_{2}\right] \ni \beta$, i.e. with the exclusion of a zero Lebesgue measure set of values of $\beta$.

We are not going to prove this proposition, as it obtainable as a special case of the next one, which will be proven here.

Theorem 1 Let $Q_{a b}$ be a fillable polynomial of some overlaps (this means that $q_{a b} Q_{a b}$ is filled). Then

$$
\lim _{N \rightarrow \infty} \lim _{x \rightarrow \beta^{2} / N}\left\langle Q_{a b}\right\rangle_{x}=\left\langle q_{a b} Q_{a b}\right\rangle
$$

where the right hand side too is understood to be taken in the thermodynamic limit.

The proof requires the following 
Lemma 1 Let $\omega($.$) and \omega_{x}($.$) be the states defined respectively by the canon-$ ical partition function and by the extended one; if we consider the ensemble of index $\left\{i_{1}, . ., i_{r}\right\}$ with $r \in[1, N]$, then in the limit $x=\beta^{2} / N$ the following relation holds:

$$
\lim _{x \rightarrow \beta^{2} / N} \omega_{N, x}\left(\sigma_{i_{1}}, \ldots, \sigma_{i_{r}}\right)=\omega_{N+1}\left(\sigma_{i_{1}}, \ldots, \sigma_{i_{r}}, \sigma_{N+1}^{r}\right)+O\left(\frac{1}{N}\right) .
$$

In this statement $r$ is an exponent, not a replica index, so if $r$ is even $\sigma_{N+1}^{r}=$ 1 , while if it is odd $\sigma_{N+1}^{r}=\sigma_{N+1}$ and we emphasized the dependence on $N$ or $N+1$ in the two averages for clarity.

Proof For the sake of simplicity let us put $\underline{\sigma}=\sigma_{i_{1}} \ldots \sigma_{i_{r}}$, and for $x=\beta^{2} / N$ we have

$$
\omega_{x=\beta^{2} / N}(\underline{\sigma})=\frac{1}{Z_{N}(\beta)} \sum_{\sigma} \exp \left(\frac{\beta}{\sqrt{N}}\left(\sum_{i<j}^{1, N} J_{i j} \sigma_{i} \sigma_{j}+\sum_{i} J_{i} \sigma_{i}\right)\right) \underline{\sigma} .
$$

Introducing a sum over $\sigma_{N+1}$ at the numerator and at the denominator (which is the same as multiplying and dividing by $2^{N}$ because there is no dependence on $\sigma_{N+1}$ ), and making the transformation $\sigma_{i} \rightarrow \sigma_{i} \sigma_{N+1}$, the variable $\sigma_{N+1}$ appears at the numerator and it is possible to construct the state $\omega$ for $N+1$ particles:

$$
\omega_{N, x=\beta^{2} / N}(\sigma)=\omega_{N+1}\left(\sigma \sigma_{N+1}^{r}\right)+O\left(\frac{1}{N}\right) .
$$

Proof of Theorem 1 A fillable polynomial will be taken of the form

$$
Q_{a b}=\sum_{i j} \frac{\sigma_{i}^{a} \sigma_{j}^{b}}{N^{2}} Q_{i j}(\sigma),
$$

so that the $a, b$ are the non-filled replicas, fillable by $q_{a b}$, and the filled replicas are included in the $Q_{i j}$ 's. We have

$$
\left\langle Q_{a b}\right\rangle_{t}=\frac{1}{N^{2}} \mathbb{E}\left[\sum_{i j} \Omega_{t}\left(\sigma_{i}^{a} \sigma_{j}^{b} Q_{i j}(\sigma)\right)\right]=\frac{1}{N^{2}} \mathbb{E}\left[\sum_{i j} \omega_{t}\left(\sigma_{i}^{a}\right) \omega_{t}\left(\sigma_{j}^{b}\right) \Omega_{t}\left(Q_{i j}\right)\right] .
$$

We will now take $x=\beta^{2} / N$ and use the previous lemma to get

$$
\omega_{x=\beta^{2} / N}\left(\sigma_{i}^{a}\right)=\omega\left(\sigma_{i}^{a} \sigma_{N+1}^{a}\right)+O\left(\frac{1}{N}\right)
$$


while the remaining part product state $\Omega_{x}$ continues to act on an even number of each replica and it is not modified:

$$
\Omega_{x=\beta^{2} / N}\left(Q_{i j}\right)=\Omega\left(Q_{i j}\right)
$$

Hence

$$
\omega\left(\sigma_{i}^{a} \sigma_{N+1}^{a}\right) \omega\left(\sigma_{i}^{b} \sigma_{N+1}^{b}\right) \Omega\left(Q_{i j}\right)=\Omega\left(\sigma_{i}^{a} \sigma_{j}^{b} \sigma_{N+1}^{a} \sigma_{N+1}^{b} Q_{i j}\right) .
$$

Using the dummy summation variable $\sigma_{N+1}$ [5] we can also sum over all dumb indexes from 1 to $N$ and divide by $N$, because the involved terms are of order $O\left(\frac{1}{N}\right)$ and become irrelevant in the $N \rightarrow \infty$ limit. Therefore

$$
\left\langle Q_{a b}\right\rangle_{x=\beta^{2} / N}=N^{-3} \mathbb{E}\left[\sum_{i j k} \Omega\left(\sigma_{k}^{a} \sigma_{k}^{b} \sigma_{i}^{a} Q_{i j} \sigma_{j}^{b}\right)\right]+O\left(\frac{1}{N}\right)
$$

and in the thermodynamic limit we complete the proof.

If we now let $x \rightarrow 0^{+}$as $\beta^{2} / N, N \rightarrow \infty$ we immediately get

Corollary 1 Let $\beta>\beta_{c}$, then

$$
\lim _{N \rightarrow \infty}\langle q\rangle_{x=\beta^{2} / N}>0
$$

Proof This is the simple case of the fillable polynomial $Q=q$ in the previous theorem, which offers $\lim _{N \rightarrow \infty}\langle q\rangle_{x=\beta^{2} / N}=\lim _{N \rightarrow \infty}\left\langle q^{2}\right\rangle \geq 0$, which becomes a strict inequality below the critical point.

The corollary shows that the the non-negativity of the average of the overlap can be easily proven with a simple cavity argument with no need of the Ghirlanda-Guerra relations, which are instead needed to prove the non-negativity of the overlap with probability one [1].

We are interested in studying the behavior of the overlap at the critical point, where the overlap begins to fluctuate. In order to do so, we will have to pay a price: we need to provide a more involved proof of an even weaker statement than that of the previous corollary. This will pave the way to a new proof of the corollary which also offers a control of the critical behavior of the overlap. 


\subsection{The expansion and the spin-flip symmetry break- ing}

We are interested in obtaining an expansion of the overlap $\left\langle q_{12}\right\rangle$ in a neighborhood of the critical inverse temperature $\beta_{c}$.

The right way to perform the expansion is indicated by the next

Proposition 4 Let $\phi_{s}$ be measurable with respect to the $\sigma$-algebra $\mathcal{A}_{s}$ generated by the overlaps among s replicas, then the following streaming equation for $\phi_{s}$ holds:

$$
\partial_{x}\left\langle\phi_{s}\right\rangle_{x}=N\left\langle\phi_{s}\left(\sum_{a, b} q_{a, b}-s \sum_{a=1}^{s} q_{a, s+1}+\frac{1}{2} s(s+1) q_{s+1, s+2}\right)\right\rangle_{x}
$$

where all the averages are considered at any given size of the system $N$.

This proposition is a straightforward generalization of the streaming equation proved in [6]. Throughout the section all the averages are understood to be taken at fixed finite $N$. If we use the proposition above in the simple case $\phi=q$, we obtain immediately

$$
\partial_{x}\left\langle q_{12}\right\rangle_{x}=N\left\langle q_{12}^{2}-4 q_{12} q_{23}+3 q_{12} q_{34}\right\rangle_{x}
$$

which at $x=0$ reduces to $N\left\langle q_{12}^{2}\right\rangle$ (with a violation of Ghirlanda-Guerra identities [4] in the thermodynamic limit) and explodes as $N \rightarrow \infty$, while it remains finite when $x>0$. We will get back to this later on.

It is from the above identity (2) that we will deduce a weaker form of Corollary 1, as stated in the next

Theorem 2 Let $x=y^{2} / N$ and $\beta>\beta_{c}$. Then, for sufficiently small but non-vanishing $y$

$$
\lim _{N \rightarrow \infty}\left\langle q_{12}\right\rangle_{x=y^{2} / N}>0
$$

Proof We are going to give place to an iterated expansion, about the critical point $\beta_{c}$, by means of a repeated use of Proposition 4, relying also 
on Proposition 3 The right hand side of (2) consists of three terms. The first term is (the average of) the filled polynomial $\left\langle q_{12}^{2}\right\rangle_{x}$, which, as we noticed, does not depend on $x$ and hence

$$
\left\langle q_{12}\right\rangle_{x}=N\left(\left\langle q_{12}^{2}\right\rangle x-4 \int_{0}^{x} d x^{\prime}\left\langle q_{12} q_{23}\right\rangle_{x^{\prime}}+3 \int_{0}^{x} d x^{\prime}\left\langle q_{12} q_{34}\right\rangle_{x^{\prime}}\right) .
$$

We want to show that the two terms under integral are of order higher than the first in $x$. We will perform this task for the first term (the case of the second is analogous) using again Proposition 4.

$$
\partial_{x}\left\langle q_{12} q_{23}\right\rangle_{x}=N\left\langle q_{12} q_{23} q_{13}\right\rangle_{x}+O\left(x^{3}\right) .
$$

Recalling the mentioned properties of filled polynomials, we immediately see that equation (4) gives

$$
\left\langle q_{12} q_{23}\right\rangle_{x}=N \int_{0}^{x}\left\langle q_{12} q_{23} q_{13}\right\rangle_{x^{\prime}} d x^{\prime}=N\left\langle q_{12} q_{23} q_{13}\right\rangle x .
$$

so that consequently

$$
\int_{0}^{x} d x^{\prime}\left\langle q_{12} q_{23}\right\rangle_{x^{\prime}}=\int_{0}^{x} d x^{\prime} N\left\langle q_{12} q_{23} q_{13}\right\rangle_{x^{\prime}} x^{\prime}+O\left(x^{3}\right) .
$$

Therefore the leading contribution from $\left\langle q_{12} q_{23}\right\rangle_{x}$ in (3) is $\left\langle q_{12} q_{23} q_{13}\right\rangle N^{2} x^{2}$, i.e. a contribution of order two in $x$. An identical consideration tells us that the contribution from the last term in equation (3) is of three in $x$. Neglecting terms of order $x^{4}$ or higher one thus gets

$$
\begin{aligned}
\left\langle q_{12}\right\rangle_{x} & =N\left\langle q_{12}^{2}\right\rangle x-2 N^{2}\left\langle q_{12} q_{23} q_{13}\right\rangle x^{2}- \\
& -\frac{4}{3} N^{3}\left\langle q_{12}^{2} q_{23}^{2}\right\rangle x^{3}+N^{3}\left\langle q_{12}^{2} q_{34}^{2}\right\rangle x^{3}+6 N^{3}\left\langle q_{12} q_{23} q_{34} q_{14}\right\rangle x^{3}+\cdots
\end{aligned}
$$

Notice that for a given strictly positive $x$, the above expansion diverges below the critical temperature (we will comment further on this at the end of the section) as $N \rightarrow \infty$, so that such an expansion makes sense only if $x \rightarrow 0^{+}$as $N \rightarrow \infty$ (this is true within our spin-flip symmetry breaking approach, and we will see later how to bypass this restriction studying the 
gauge symmetry breaking). Let hence put $x=y^{2} / N$, as announced. Then we can rewrite the previous expansion (5) as

$$
\begin{aligned}
\left\langle q_{12}\right\rangle_{x}=\left\langle q_{12}^{2}\right\rangle y^{2} & -2\left\langle q_{12} q_{23} q_{13}\right\rangle y^{4}- \\
& -\frac{4}{3}\left\langle q_{12}^{2} q_{23}^{2}\right\rangle y^{6}+\left\langle q_{12}^{2} q_{34}^{2}\right\rangle y^{6}+6\left\langle q_{12} q_{23} q_{34} q_{14}\right\rangle y^{6}+\cdots .
\end{aligned}
$$

For sufficiently small $y$ the sign of the left hand side is determined by the first term in the expansion, which is zero above the critical temperature but strictly positive below (for otherwise the replica symmetric solution would hold at low temperatures, which is not possible. See [12, 7] for details). Hence

$$
\lim _{N \rightarrow \infty}\left\langle q_{12}\right\rangle_{x=y^{2} / N}>0, \beta>\beta_{c},
$$

while we already emphasized that

$$
\lim _{N \rightarrow \infty}\left\langle q_{12}\right\rangle_{x=0} \equiv 0 .
$$

In other words, the limit depends on the way it is performed, and a different way to identify the transition is stating that the two limits $x \rightarrow 0^{+}$and $N \rightarrow \infty$ (which we take simultaneously) cannot be interchanged below the critical temperature. Looking at (2) - and its consequent expansion (5) - we can see that this transition we just proved is connected with the violation of Ghirlanda-Guerra identities [4, when $\beta>1$ and $x=0$ vs $x>0$ (we will deepen this further in a work still in progress at the moment).

\section{Critical indices}

We saw that, due to the gauge symmetry, the choice $x=\beta^{2} / N$ is equivalent to the addition of a new spin, say numbered $N+1$ (see for instance Lemma 11). So could we choose in the previous section $y=\beta$ (which makes the external field a proper cavity field), we would in some sense identify the transition by getting two different results when performing the limit as $N \rightarrow \infty$ of the average of the overlap for $N$ or $N+1$ spins. Unfortunately 
in the previous section $y$ has to be small enough and this interpretation in general is not possible within the proof we provided. Still, the expansion we illustrated can be adapted in such a way to recover the same result not limited to small $y$, and to obtain the critical exponents too.

Roughly speaking, the idea is that even if $y$ is not necessarily small, the overlaps will be small anyways, as they are always bounded by one, and they are identically zero above the critical temperature, at which they start deviating from zero with continuity. This fact, together with an explicit use of the gauge symmetry (as opposed to mere spin-flip) will allow for the reformulation of the expansion we need.

\subsection{Lower bound for the first critical index}

Critical indices are needed to characterize singularities of the theory at the critical point and are related to the overlap correlation functions.

Let us introduce the expansion parameter

$$
\tau=\left(\beta^{2}-1\right) / 2
$$

and let us focus on the averaged squared overlap $\left\langle q_{12}^{2}\right\rangle$. Assuming continuity in $\beta$ at the critical point, let us also assume a behavior of the form

$$
\left\langle q_{12}^{2}\right\rangle \sim C \tau^{\gamma}
$$

Once $\gamma$ is known, we can get all the other critical exponents for the higher order correlation functions.

We will use convexity of the free energy density to obtain a bound for $\gamma$ at a given $C$. We know that

$$
\partial_{\beta}^{2} \alpha_{N}(\beta) \geq 0
$$

which means that the internal energy density $u_{N}(\beta)=-\partial_{\beta} \alpha_{N}(\beta)$ is a decreasing function of $\beta$

$$
\partial_{\beta} u_{N}(\beta) \leq 0
$$


Therefore for every $\beta>1$, we have $u(\beta) \leq u(1)$. From now on we will restrict ourselves to the case $\beta>1$. We can thus write

$$
-\frac{\beta}{2}\left(1-\left\langle q_{12}^{2}\right\rangle\right) \leq-\frac{1}{2}\left(1-\left.\left\langle q_{12}^{2}\right\rangle\right|_{\beta=1}\right)=-\frac{1}{2}
$$

so that

$$
\left\langle q_{12}^{2}\right\rangle \leq \frac{\beta-1}{\beta} .
$$

Using our assumption $\left\langle q_{12}^{2}\right\rangle=C \tau^{\gamma}+o\left(\tau^{\gamma}\right)$, the previous inequality (6) reads

$$
C \tau^{\gamma}+o\left(\tau^{\gamma}\right)<\tau+o(\tau)
$$

Now,

- if $\gamma<1$, condition (7) is not fulfilled. In fact, if we divide by $\tau^{\gamma}$, the condition reads $C \leq \tau^{1-\gamma}+o(1)$, and since $1-\gamma>0$, in the limit $\tau \rightarrow 0$ we would get $C \leq 0$, which is not possible;

- if $\gamma=1$, condition (7) becomes $C \leq 1+o(1)$, which in the limit $\tau \rightarrow 0$ gives $C \leq 1$.

Hence $\gamma \geq 1$, and in particular if $\gamma=1$ then $0<C<1$. We are about to prove that $\gamma=2$.

\subsection{A revisited variational approach}

Let $p$ be a generic filled overlap polynomial. With an abuse of notation, we follow [2] and consider the pressure, expanded in overlap polynomials, as a function of the polynomials with an explicit dependence on the inverse temperature: $\alpha(\beta)=\alpha(\langle p(\beta)\rangle, \beta)$. More precisely (see [2])

$$
\alpha(\beta,\langle p(\beta)\rangle)=\ln 2+\frac{\beta^{2}}{4}\left[1+\left(1-\beta^{2}\right)\left\langle q_{12}^{2}\right\rangle\right]+\frac{\beta^{6}}{3}\left\langle q_{12} q_{23} q_{13}\right\rangle+O\left(\beta^{8}\right)
$$

and we are interested in computing its derivative with respect to $\beta^{2}$. As explained in details in [2], the total derivative of the $\alpha(\beta)$ with respect to $\beta^{2}$ is proportional to the internal energy of the model, and as $\alpha(\beta)=-\beta f(\beta)$, 
where $f(\beta)$ is the free energy density, we derive with respect to $\beta^{2}$ rather than to $\beta$. This is just matter of tastes, after all, because it would change just a factor in front of our expressions below. We have

$$
\frac{d \alpha(\beta,\langle p(\beta)\rangle)}{d \beta^{2}}=\frac{1}{2 \beta} \frac{d \alpha(\beta,\langle p(\beta)\rangle)}{d \beta}=\frac{1}{4}\left(1-\left\langle q_{12}^{2}\right\rangle\right)
$$

and this expression needs to be equal to

$$
\frac{d \alpha(\beta,\langle p(\beta)\rangle)}{d \beta^{2}}=\partial_{\beta^{2}} \alpha(\beta,\langle p(\beta)\rangle)+\sum_{p} \frac{\partial \alpha(\beta,\langle p(\beta)\rangle)}{\partial\langle p(\beta)\rangle} \frac{\partial\langle p(\beta)\rangle}{\partial \beta^{2}} .
$$

By means of some tedious calculations that we will not report (we rather refer the interested reader to [2]), we get

$$
\partial_{\beta^{2}} \alpha(\beta,\langle p(\beta)\rangle)=\frac{1}{4}\left(1-\left\langle q_{12}^{2}\right\rangle\right),
$$

which imposes the following constraint

$$
\sum_{p} \frac{\partial \alpha(\langle p\rangle, \beta)}{\partial\langle p\rangle} \frac{\partial\langle p\rangle}{\partial \beta^{2}}=0
$$

where the sum on $p$ denotes the sum over all the overlap correlation functions appearing in the expansion. From this equation, one can deduce [2] the Aizenman-Contucci polynomials (AC) through an approach different from stochastic stability (see [1]). Here we want to employ such an approach to derive all the critical exponents of the theory within an iterative scheme.

Let us take the first two non trivial orders (with $\left\langle q_{12}^{2}\right\rangle,\left\langle q_{12} q_{23} q_{13}\right\rangle$ ) and write, neglecting terms of higher order than $O\left(q^{4}\right)$

$$
\frac{\partial \alpha(\beta)}{\partial\left\langle q_{12}^{2}\right\rangle} \frac{\partial\left\langle q_{12}^{2}\right\rangle}{\partial \beta^{2}}+\frac{\partial \alpha(\beta)}{\partial\left\langle q_{12} q_{23} q_{13}\right\rangle} \frac{\partial\left\langle q_{12} q_{23} q_{13}\right\rangle}{\partial \beta^{2}}=0 .
$$

From (3) and the first AC relation

$$
\left\langle q_{12}^{4}\right\rangle-4\left\langle q_{12}^{2} q_{23}^{2}\right\rangle+3\left\langle q_{12}^{2} q_{34}^{2}\right\rangle=0
$$

we get, when $x=\beta^{2} / N$,

$$
\begin{aligned}
\left\langle q_{12}\right\rangle & =\left\langle q_{12}^{2}\right\rangle \beta^{2}-2\left\langle q_{12} q_{23} q_{13}\right\rangle \beta^{4}-\frac{1}{3}\left\langle q_{12}^{4}\right\rangle+6\left\langle q_{12} q_{23} q_{34} q_{14}\right\rangle \beta^{6}+O\left(q^{5}\right) \\
\left\langle q_{12} q_{23}\right\rangle & =\left\langle q_{12} q_{23} q_{13}\right\rangle \beta^{2}+\left\langle q_{12}^{2} q_{23}^{2}\right\rangle \beta^{4}-3\left\langle q_{12} q_{23} q_{34} q_{14}\right\rangle \beta^{6}+O\left(q^{5}\right) .
\end{aligned}
$$


Recalling Proposition 1 we also obtain

$$
\begin{aligned}
\left(\beta^{2}-1\right)\left\langle q_{12}^{2}\right\rangle & =2 \beta^{4}\left\langle q_{12} q_{23} q_{13}\right\rangle+\frac{\beta^{6}}{3}\left\langle q_{12}^{4}\right\rangle-6 \beta^{6}\left\langle q_{12} q_{23} q_{34} q_{14}\right\rangle(9) \\
\left(\beta^{2}-1\right)\left\langle q_{12} q_{23} q_{13}\right\rangle & =-\beta^{4}\left\langle q_{12}^{2} q_{23}^{2}\right\rangle+3 \beta^{4}\left\langle q_{12} q_{23} q_{34} q_{14}\right\rangle .
\end{aligned}
$$

At this point the $\mathrm{AC}$ relations are not enough, and we need their non-linear extension expressed in the following Ghirlanda-Guerra identities [4]

$$
\left\langle q_{12}^{2} q_{13}^{2}\right\rangle=\frac{1}{2}\left\langle q_{12}^{4}\right\rangle+\frac{1}{2}\left\langle q_{12}^{2}\right\rangle^{2},\left\langle q_{12}^{2} q_{34}^{2}\right\rangle=\frac{1}{3}\left\langle q_{12}^{4}\right\rangle+\frac{2}{3}\left\langle q_{12}^{2}\right\rangle^{2},
$$

to deduce from (91)-(10) the expression of the overlap correlation functions of higher orders in terms of the two-replica overlap

$$
\begin{aligned}
\beta^{2}\left\langle q_{12} q_{23} q_{13}\right\rangle & =\tau\left\langle q_{12}^{2}\right\rangle+\frac{1}{3}\left\langle q_{12}^{4}\right\rangle+\frac{1}{2}\left\langle q_{12}^{2}\right\rangle^{2}+O\left(q^{5}\right), \\
\left\langle q_{12} q_{23} q_{34} q_{14}\right\rangle & =\frac{1}{6}\left\langle q_{12}^{4}\right\rangle+\frac{1}{6}\left\langle q_{12}^{2}\right\rangle^{2}+\frac{2}{3} \tau^{2}\left\langle q_{12}^{2}\right\rangle+O\left(q^{5}\right) .
\end{aligned}
$$

Now, calling $\left\langle q_{12}^{2}\right\rangle=m^{2}(\beta)$ and noting that $\left\langle q_{12} q_{23} q_{13}\right\rangle \sim \tau m^{2}(\beta) \sim$ $C \tau^{\gamma+1}$, we substitute $m^{2}(\beta)$ into (8) and get

$$
-\frac{\tau}{2} \frac{d\left(m^{2}\right)}{d \beta^{2}}+\frac{1}{3} \frac{d\left(\tau m^{2}\right)}{d \beta^{2}}=0
$$

where the factors $\frac{1}{2}$ and $\frac{1}{3}$ come from the form of the expansion of the free energy (see [2] for details). The derivative of second term in the left hand side above gives

$$
\frac{d\left(\tau m^{2}\right)}{d \beta^{2}}=\frac{1}{2} m^{2}+\tau \frac{d\left(m^{2}\right)}{d \beta^{2}},
$$

so that we obtain a differential equation trivially solvable by separation of variables:

$$
\frac{d \beta^{2}}{\tau(\beta)}=\frac{d m^{2}}{m^{2}}=d \log \left(m^{2}(\beta)\right) .
$$

Integrating against $d\left(\beta^{\prime}\right)^{2}$ yields

$$
\int_{\beta_{1}^{2}}^{\beta^{2}} \frac{2 d\left(\beta^{\prime}\right)^{2}}{\beta^{2}-1}=\int_{\beta_{1}^{2}}^{\beta^{2}} d \log m^{2}(\beta) \rightarrow \log \left(\beta^{2}-1\right)^{2}=\log \left(m^{2}(\beta)\right)+C
$$

where $\beta^{2}>\beta_{1}^{2}>1$ and $C$ will depend on $\beta_{1}$. So we finally have

$$
\left\langle q_{12}^{2}\right\rangle=m^{2}(\beta)=C\left(\beta^{2}-1\right)^{2}+O\left(\left(\beta^{2}-1\right)^{3}\right) \sim C \tau^{2} .
$$


Therefore the critical index for the first overlap correlation function is $\gamma=$ 2. From this, we immediately deduce

$$
\begin{aligned}
\left\langle q_{12} q_{23} q_{13}\right\rangle & \sim C \tau^{3} \\
\left\langle q_{12} q_{23} q_{34} q_{14}\right\rangle & \sim C^{\prime} \tau^{4} \\
\left\langle q_{12}^{4}\right\rangle & \sim C^{\prime \prime} \tau^{4}
\end{aligned}
$$

and so on.

Notice that the steps we illustrated in this section provide an improvement of Theorem 2 as they allow to extend its statement up to $y=\beta$, thus recovering Corollary 1 .

\section{Conclusions and Outlook}

In this paper we have shown that the SK model exhibits the phase transition predicted by Parisi in terms of the discontinuous response of the overlap to the presence of and external field. A simple iterated power expansion of the overlaps within the cavity method allowed for the proof of the results. The techniques we used are extensions of those introduced and employed in [2] and [3]. As a result the control near the critical point of the overlap discontinuity in the $(\beta, x)$ plane follows, together with the computation of the critical exponents of the overlap correlation functions.

\section{Acknowledgment}

The authors are pleased to thank Francesco Guerra for his constant priceless scientific support. We are also very grateful to Peter Sollich, Silvio Franz and Andrea Pagnani for fruitful discussions. The authors thank an anonymous referee for useful suggestions. 


\section{References}

[1] M. Aizenman, P. Contucci, On the stability of the quenched state in mean field spin glass models, J. Stat. Phys. 92 765-783 (1998).

[2] A. Barra, Irreducible free energy expansion for mean field spin glass model, J. Stat. Phys. 123-3 601-614 (2006).

[3] A. Barra, L. De Sanctis, Overlap Fluctuations from Random Overlap Structures, ArXiv:cond-mat/0607615, to appear slightly simplified as Overlap Fluctuations from the Boltzmann Random Overlap Structure in J. Math. Phys. 47103305 (2006)

[4] S. Ghirlanda, F. Guerra, General properties of overlap distributions in disordered spin systems. Towards Parisi ultrametricity, J. Phys. A 31 9149-9155 (1998).

[5] F. Guerra, Fluctuations and thermodynamic variables in mean field spin glass models, in "Stochastic provesses, physics and geometry, II", S. Albeverio et al. eds., Singapore (1995).

[6] F. Guerra, Sum rules for the free energy in the mean field spin glass model, in Mathematical Physics in Mathematics and Physics: Quantum and Operator Algebraic Aspects, Fields Institute Communications 30, American Mathematical Society (2001).

[7] F. Guerra, The replica symmetric region in the SherringtonKirkpatrick mean field spin glass model. The Almeida-Thouless line, ArXiv:cond-mat/0604674, to appear in J. Stat. Phys..

[8] E. Marinari, G. Parisi, J. Ruiz-Lorenzo, F. Ritort Numerical evidence for spontaneously broken replica symmetry in $3 D$ spin glasses, Phys. Rev. Lett. 92 (1996) .

[9] M. Mézard, G. Parisi and M.A. Virasoro, Spin glass theory and beyond, World Scientific, Singapore (1987). 
[10] G. Parisi, Magnetic Properties of Spin Glasses in a new mean field theory, J. Phys. A: Math. Gen. 1887-1895 (1980).

[11] M. Talagrand, Spin glasses: a challenge for mathematicians. Cavity and Mean field models, Springer Verlag (2003).

[12] F.L. Toninelli, About the Almeida-Thouless transition line in the Sherrington-Kirkpatrick mean field spin glass model, Europhys. Lett. 60 (2002). 